\title{
Goldberg-Shprintzen megacolon syndrome
}

INSERM

\section{Source}

INSERM. (1999). Orphanet: an online rare disease and orphan drug data base. GoldbergShprintzen megacolon syndrome. ORPHA:66629

A rare multiple cong enital anomalies/dysmorphic syndrome characterized by Hirschsprung disease, facial dysmorphism (sloping forehead, high arched eyebrows, long eyelashes, telecanthus/hypertelorism, ptosis, prominent ears, thick earlobes, prominent nasal bridge, thick philtrum, everted lower lip vermillion and pointed chin), global developmental delay, intellectual disability and variable cerebral abnormalities (focal or generalized polymicrogyria, or hypoplastic corpus callosum). 